\title{
Mesenchymal Chondrosarcoma of the Infratemporal Fossa-A Rare Case Report
}

\author{
Shalender Sharma1*, J. K. Dayashankara Rao', Harsh Jain², Kaberi Majumder3, \\ Neelima Gehlot ${ }^{1}$ \\ ${ }^{1}$ Department of Oral and Maxillofacial Surgery, SGT Dental College, Gurgaon, India \\ ${ }^{2}$ Department of Oral and Maxillofacial Surgery, Sharada Dental College, Greater Noida, India \\ ${ }^{3}$ Department of Orthodontics, SGT Dental College, Gurgaon, India \\ Email: ${ }^{\text {Sharma.shalender@rediffmail.com }}$
}

Received 5 January 2014; revised 4 February 2014; accepted 3 March 2014

Copyright (C) 2014 by authors and Scientific Research Publishing Inc.

This work is licensed under the Creative Commons Attribution International License (CC BY).

http://creativecommons.org/licenses/by/4.0/

(c) (i) Open Access

\begin{abstract}
Chondrosarcoma is a malignant neoplasm that results in abnormal bone and cartilage growth. Although Chondrosarcoma is rare, it is the second most common primary bone malignancy. Mesenchymal Chondrosarcoma (MCS) is a rare histological variant of Chondrosarcoma (CS) that accounts for only $1 \%$ of all CS and has high predilection for the head and neck region with a peak incidence in $2^{\text {nd }}$ and $3^{\text {rd }}$ decade of life. There is comparable male to female ratio with two thirds of the cases arising from bone and one third from soft tissue. The tumor is unique because of its aggressive growth with a high tendency for late recurrence and delayed metastasis. We present a case of this highly malignant lesion arising from the infratemporal fossa and rapidly involving maxillary tuberosity and mandibular ramus.
\end{abstract}

\section{Keywords}

Chondrosarcoma; Mesenchymal Chondrosarcoma; Bone Neoplasm

\section{Introduction}

Mesenchymal Chondrosarcoma (MCS) is a rare tumour characterized by undifferentiated mesenchymal cells with islands of mature hyaline cartilage. Lichtenstein and Bernstein first described bony MCS in 1959. Dowling subsequently reported this entity in the soft tissue in 1964 [1].

MCS occurs most often in the second and third decades of life. It can occur anywhere in the body, both in skeletal and extra skeletal sites, including the orbit, viscera, meninges, or brain. However, it tends to occur 2 to 3

${ }^{*}$ Corresponding author. 
times more commonly in bones than in extra skeletal sites. In a review of 111 cases, 72 tumours occurred in skeletal sites, 22 of which affected the craniofacial bones; 38 tumours occurred in extra skeletal sites, with only 1 patient having orbital soft tissue involvement; and 1 occurred in both skeletal and extra skeletal sites [2]. It has a variable clinical course with frequent recurrence and occasional distant osseous and visceral metastatic spread. The prognosis is poor, independent of the type of treatment [3]. We are presenting a case report of MCS of infratemporal fossa in a young female which has involved maxillary tuberosity, ramus of the mandible and extending towards the temporal bone.

\section{Case Report}

A 23-year-old female reported in the OPD of oral surgery department with the complaint of swelling on the left side of the face since past 3 months. History revealed that the swelling is increasing progressively in size over the period of 3 months. Patient had restricted mouth opening and pain in the left maxillary tuberosity area since last 15 days. On examination a diffuse swelling was seen causing facial asymmetry on the left side of the face ovoid in shape measuring $8 \times 6 \mathrm{~cm}$ in size. The swelling was firm in consistency, fixed to the underlying bone and non tender on palpation (Figure 1). No secondary changes of ulcer or sinus were seen and there was no sign of pus discharge. Intraorally no ulcerative growth was present. Expansion of the palatal as well as buccal cortical plate was present in the areas w.r.t 27 and 28 region. A provisional diagnosis of malignant bony neoplasm was given (Figure 1). Panoramic radiography revealed decreased radiodensity in the left maxillary tuberosity and ramus area. There was a typical sunburst appearance in the infratemporal area involving maxillary tuberosity, infratemporal bone and mandibular ramus area (Figure 2). CT scan revealed osteosclerosis and erosion of the

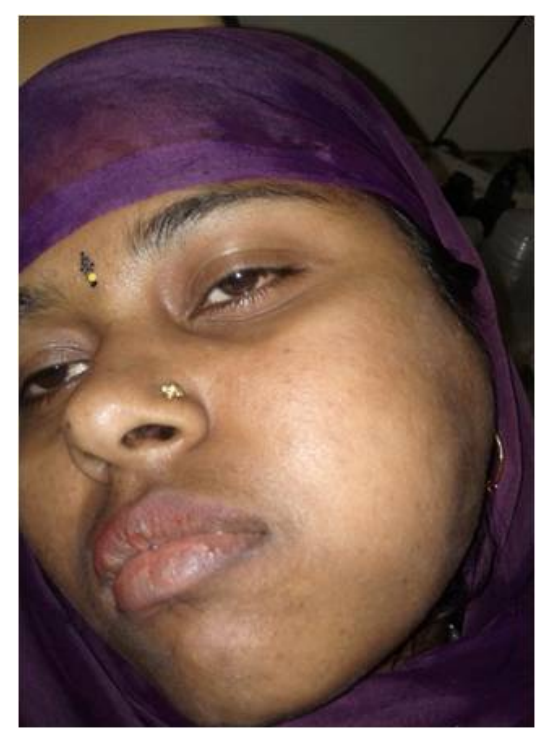

Figure 1. Extra oral view of the swelling.

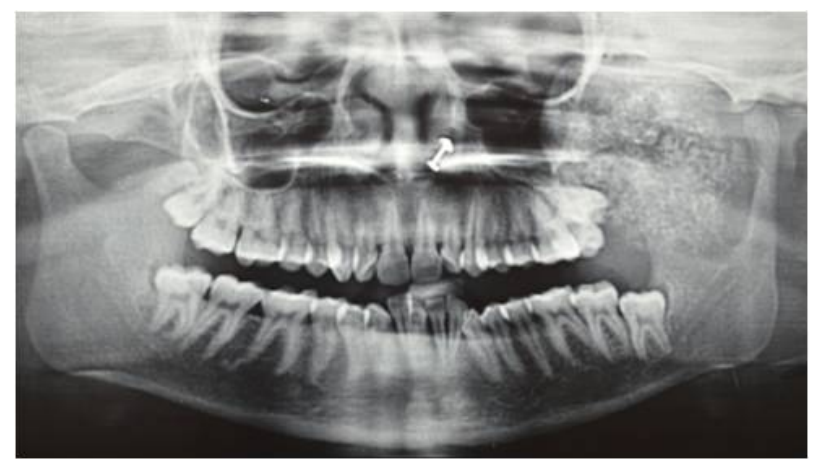

Figure 2. OPG showing mass in infratemporal region. 
whole infratemporal fossa involving maxillary tuberosity, infratemporal surface of temporal bone and mandibular ramus area. A nonenhancing mass with mottled calcification was seen in the axial section measuring $5 \times 5$ $\mathrm{cm}$ in size. The classic sunburst appearance is also seen (Figures 3 and 4). An incisional biopsy was then done. Histologic examination revealed a biomorphic tumor displaying areas of well differentiated cellular hyaline cartilage surrounded by small undifferentiated tumor cells that exhibit a hemangiopericytoma like pattern of arrangement (Figure 5). The cartilaginous areas show prominent calcification with focal evidence of ossification. Tumor infiltration into bone is noted. Immunohistochemistry was done and the chondrocytes were positive for S100. Histopathology was suggestive of MS. The patient underwent surgery and chemotherapy.

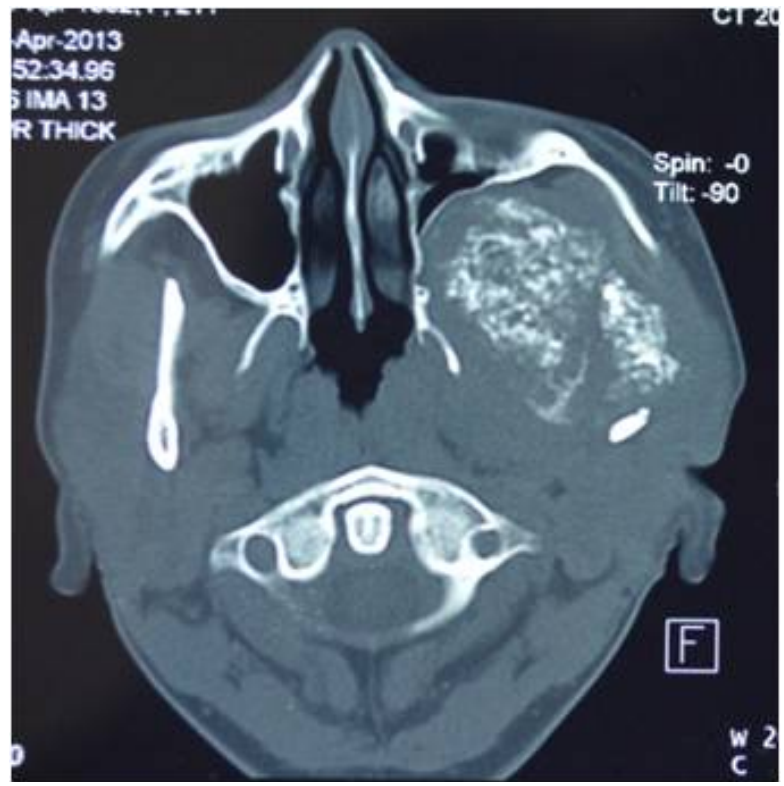

Figure 3. CT axial view showing big mass in the infratemporal region.

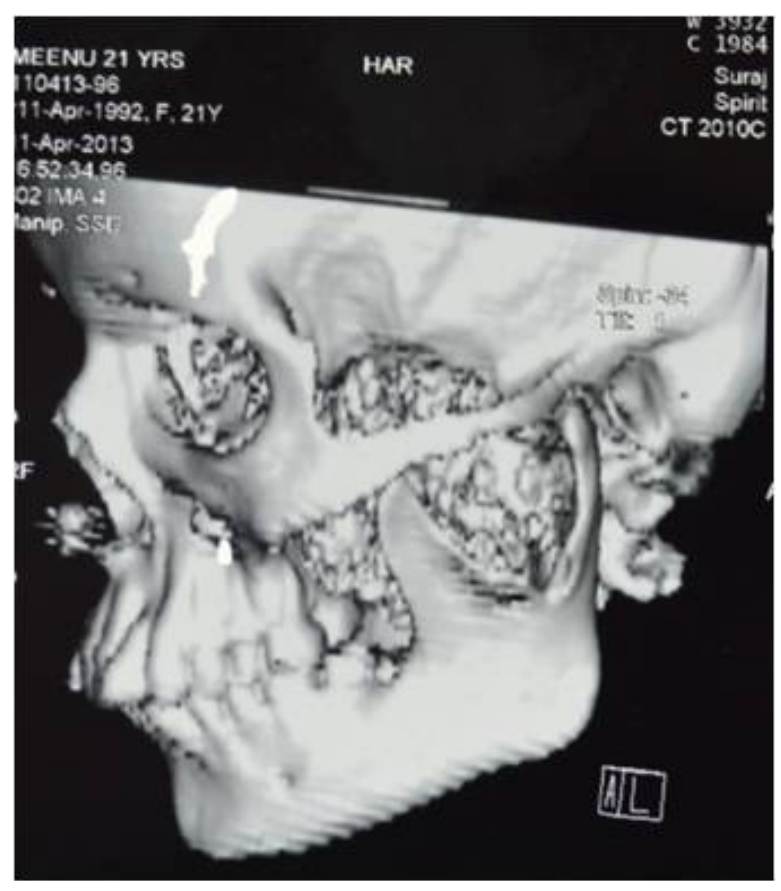

Figure 4. 3D CT image of the lesion in infratemporal region. 


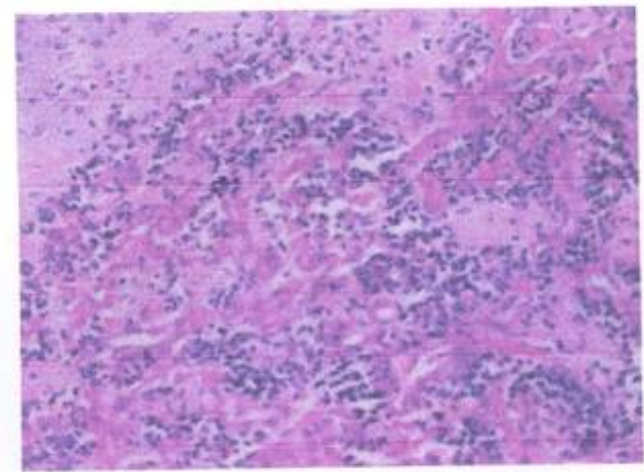

Figure 5. Photomicrograph of the specimen suggestive of mesenchymal chondrosarcoma.

\section{Discussion}

Phemister in 1930 defined Chondrosarcoma as sarcomas of bone containing abundant cartilage. Lichtenstein and Jaffe established criteria for classification of Chondrosarcoma. They defined Chondrosarcoma as arising from fully fledged cartilage and never containing osteoid or bone stroma [4]. According to international classification of diseases for oncology [5], CS has been classified into six subtypes, on the basis of the tumor location, the histological characteristics of the malignant cartilage cells and the makeup of the surrounding matrix material associated with the tumor are as follows: 1) Chondrosarcoma NOS (Not otherwise specified, 2) Juxtacortical Chondrosarcoma, 3) Myxoid Chondrosarcoma, 4) Mesenchymal Chondrosarcoma, 5) Clear cell Chondrosarcoma, 6) Dedifferentiated Chondrosarcoma.

MCS is a rare entity originally described by Lichtenstein and Bernstein as a biphasic tumour consisting of spindle cell mesenchyme mixed with areas of chondroid differentiation accounting for only about $1 \%$ of all Chondrosarcoma [4]. It develops from a pluripotent mesenchymal stem cell which can differentiate into angioblastic, fibroblastic and cartilaginous structures.

Approximately one-third of MCS are found outside bone within a variety of soft tissues, while two-thirds of these tumors are intraosseous. Most chondrosarcomas of the head and neck region occur in the maxilla; others are seen to occur less commonly in the body of the mandible, the ramus, the nasal septum and the paranasal sinuses [6]. In the maxilla, the most common location is anterior alveolus where preexisting nasal cartilage is present. Our case is one of the rare cases because it is occurring in the infratemporal fossa and involves maxillary tuberosity, infratemporal surface of maxilla and ramus of the mandible and extending towards the temporal bone.

MCS is more common during the second and third decades of life, therefore it is a malignancy of the young individuals as seen in our cases. There are no specific clinical signs or symptoms but the first clinical symptom is a painless swelling or mass, rapidly growing to a large size and leading to facial deformity, nasal obstruction, expansion of the bone and its subsequent perforation as it enlarges, surface ulceration may also be seen [7]. Later symptoms include pain and mobility of the teeth in the region. Rarely facial paralyses can also occur.

Radiographically, chondrosarcoma is seen as an irregularly shaped, ill-defined radiolucency with randomly scattered, opaque mottled areas. The lesion may appear lobulated and may elevate the adjacent periosteum to produce radiating laminae in a sunray pattern as seen in our case. Perforation of the cortex of the involved bone by a cartilaginous lesion is considered a sign of probable malignancy. Since these are not pathognomonic signs of chondrosarcoma the radiographic differential diagnosis also includes fibrosarcoma and osteosarcoma. Evaluation of the maxillary region on conventional radiographs is somewhat limited due to anatomical superimposition and two dimensional displays. CT performed with contrast enhancement is the imaging modality of choice because it best delineates the bony involvement while defining local invasion into the adjacent soft tissues as seen in both our reported cases [8].

Molecular pathology of mesenchymal chondrosarcoma has been evaluated with respect to differentiating it from other small cell sarcomas. Muller and co-workers [9] have found that it expresses type II collagen which differentiates it from other small cell sarcomas such as Ewing's, synovial sarcoma and haemangiopericytoma.

Our case was characteristic of MC as dense population of anaplastic cells in a chondroid matrix was seen and 
since there was marked cellular and nuclear pleomorphism, nuclear hyperchromasia; our case was considered high grade. Immunohistochemistry also can be done for difficult cases, chondroid areas are positive for S100 protein and neuron-specific enolase is focally positive for primitive mesenchymal cells. Our case was S100 positive for chondrocytes

Wide surgical excision is the mainstay of treatment for MC in the jaw bones [10]. These tumors are radioresistant and chemotherapy can be used as an adjuvant therapy after wide surgical excision is made. The benefit of neo-adjuvant modalities for MC has not been proven. However the use of preoperative radiotherapy prior to radical resection has been shown to reduce tumour bulk [11]. Postoperative radiotherapy has been reported to increase survival rates although the studies done did not have statistically significant numbers of patients [12]. The overall survival rate at 5 and 10 years is $55 \%$ and $27 \%$ respectively [2]. Our case was treated with wide surgical excision followed by chemotherapy. Generally the prognosis of $\mathrm{MC}$ is considered to be poor and the grade, the size of the tumor, the adequacy of tumor resection margins may be prognostic indictors. Local recurrence occurs and may indicate subsequent metastasis; hence adequate treatment and long-term follow-up, including periodic systemic evaluation, are required for patients with MC of the maxillofacial region.

\section{Conclusion}

We present a case of MC, because MC is an uncommon tumor and is rarely found in the jaw. An adequate biopsy is enough for diagnosis. It may be radiolucent, radio-opaque or mixed. MCS requires surgical excision with wide margins. Pre- and postoperative chemotherapy or radiation may be a choice of treatment, although their effectiveness is unclear.

\section{References}

[1] Dowling, E.A. (1964) Mesenchymal Chondrosarcoma. The Journal of Bone \& Joint Surgery, 46-A, 747-754.

[2] Nakashima, Y., Unni, K.K., Shives, T.C., et al. (1986) Mesenchymal Chondrosarcoma of Bone and Soft Tissue: A Review of 111 Cases. Cancer, 57, 2444-2453. http://dx.doi.org/10.1002/1097-0142(19860615)57:12<2444::AID-CNCR2820571233>3.0.CO;2-K

[3] Harwood, A.R., Krajbich, J.I. and Fornasier, V.L. (1981) Mesenchymal Chondrosarcoma: A Report of Ases. Clinical Orthopaedics, 158, 144-148.

[4] Ewing, J.A. (1939) Review of the Classification of Bone Tumours. Surgery, Gynecology \& Obstetrics, 40, 971-976.

[5] Percy, C., Van Holten, V. and Muir, C. (1992) International Classification of Diseases for Oncology. 2nd Edition, World Health Organization, Geneva.

[6] Anil, S., Beena, V.T., Lal, P.M. and Varghese, B.J. (1998) Chondrosarcoma of the Maxilla. Case Report. Australian Dental Journal, 43, 172-174. http://dx.doi.org/10.1111/j.1834-7819.1998.tb00160.x

[7] Tien, N., et al. (2007) Mesenchymal Chondrosarcoma of the Maxilla: Case Report and Literature Review. Journal of Oral and Maxillofacial Surgery, 65, 1260-1266. http://dx.doi.org/10.1016/j.joms.2005.11.074

[8] Vipul, J. and Sunita, G. (2011) Mesenchymal Chondrosarcoma of Maxilla: A Rare Case Report. Medicina Oral Patologia Oral y Cirugia Bucal, 16, e493-e496.

[9] Muller, S., Soder, S., Oliveira, A.M., Inwards, C.Y. and Aigner, T. (2005) Type II Collagen as Specific Marker for Mesenchymal Chondrosarcomas Compared to Other Small Cell Sarcomas of the Skeleton. Modern Pathology, 18, 1088-1094. http://dx.doi.org/10.1038/modpathol.3800391

[10] Rajan, S., Hayati, A.R.N., Ab Rahman, S. and Samsudin, A.R. (2007) Chondrosarcoma of the Mandible: A Case Report. JCDA, 73, 175-178.

[11] Knott, P.D., Gannon, F.H. and Thompson, L.D.R. (2003) Mesenchymal Chondrosarcoma of the Sinonasal Tract: A Clinicopathological Study of 13 Cases with a Review of the Literature. Laryngoscope, 113, 783-790. http://dx.doi.org/10.1097/00005537-200305000-00004

[12] Aryoshi, Y. and Shimahara, M. (1999) Mesenchymal Chondrosarcoma of the Maxilla: Report of a Case. Journal of Oral and Maxillofacial Surgery, 57, 733-737. http://dx.doi.org/10.1016/S0278-2391(99)90443-3 